\title{
Luminosity functions of gamma-ray burst afterglows
}

\author{
G. Jóhannesson, G. Björnsson, and E. H. Gudmundsson
}

\author{
Science Institute, University of Iceland, Dunhaga 3, 107 Reykjavik, Iceland \\ e-mail: gudlaugu@raunvis.hi.is
}

Received 6 June 2007 / Accepted 12 July 2007

\begin{abstract}
Aims. Use the standard fireball model to create virtual populations of gamma-ray burst afterglows and study their luminosity functions. Methods. We randomly vary the parameters of the standard fireball model to create virtual populations of afterglows. We use the luminosity of each burst at an observer's time of 1 day to create a luminosity function and compare our results with available observational data to assess the internal consistency of the standard fireball model.

Results. We show that the luminosity functions can be described by a function similar to a log normal distribution with an exponential cutoff. The function parameters are frequency dependent but not very dependent on the model parameter distributions used to create the virtual populations. Comparison with observations shows that while there is good general agreement with the data, it is difficult to explain simultaneously the X-ray and optical data. Possible reasons for this are discussed and the most likely one is that the standard fireball model is incomplete and that decoupling of the X-ray and optical emission mechanism may be needed.
\end{abstract}

Key words. gamma rays: bursts - gamma rays: theory - methods: numerical

\section{Introduction}

Since its discovery in 1997 (Costa et al. 1997; van Paradijs et al. 1997), the standard fireball model, or extensions thereof, has been used to model gamma ray burst (GRB) afterglow emission (e.g. Panaitescu 2005; Jóhannesson et al. 2006b). With dedicated follow-up observations, the number of detected afterglows has been steadily increasing. The rapid burst localisation of the Swift satellite and its on-board X-ray (XRT) telescope have substantially increased the afterglow detection rate and the number of detected afterglows is now well over $200^{1}$. This has inspired statistical investigations of afterglow properties; among which are studies exploring the luminosity function at a given observer time (Gendre \& Boër 2005; Kann et al. 2006; Liang \& Zhang 2006; Nardini et al. 2006, 2007).

Motivated by this previous work, we have created theoretical luminosity functions of GRB afterglows using the standard fireball model. We show that these luminosity functions can be described to a high degree of accuracy by an analytical function. Furthermore, we show that the parameters of that function are for the most parts independent of the chosen model parameter distributions, giving a robust estimate of the luminosity function of GRB afterglows. We also show that our theoretical luminosity function is similar to the observationally determined one, both in X-rays and optical wavebands but only if they are considered separately. It is difficult to explain the observed luminosity functions simultaneously in both wavebands. Several possible reasons for this are discussed, the most likely one being that the standard fireball model is incomplete.

The outline of this Letter is as follows: the procedure for creating the luminosity functions is described in Sect. 2 and we present our results and discuss how they depend on the model parameters in Sect. 3. Section 4 is devoted to comparison with observations and we conclude the Letter in Sect. 5.

1 see http://www.mpe.mpg.de/ jcg/grbgen.html for the most recent list.

\section{A virtual world of afterglows}

The afterglows are created numerically with the model described in Jóhannesson et al. (2006b). It is based on the standard fireball jet model, in which the energy is injected instantaneously into a narrow jet. To create the afterglows, model parameters are selected at random from pre-defined distributions (see also Jóhannesson et al. 2006a). Each parameter range is determined so that it represents results found by fitting afterglow observations with the standard fireball model or extensions thereof (e.g. Panaitescu \& Kumar 2001; Panaitescu 2005; Jóhannesson et al. 2006b). Table 1 shows the default parameter ranges used in this work. The parameter values can in many cases vary by more than an order of magnitude from one afterglow to another. All model parameters, except $p$, are therefore varied on a logarithmic scale to get a more realistic parameter distribution within the chosen range. To concentrate on the intrinsic properties of the afterglows, we fix the redshift at $z=1$.

To calculate the luminosity functions, we use the afterglow luminosity density at an observer's time of 1 day post-burst. In fact, any time can be used, but this choice is well suited for comparison with observations (Liang \& Zhang 2006; Nardini et al. 2006). One day after the burst is also late enough in the afterglow evolution so that both the optical and X-ray emission can be assumed to originate in the forward shock of the standard fireball model (Nousek et al. 2006). To reduce statistical errors, each virtual world consists of 50000 afterglows, resulting in statistical errors of the order of $1 \%$ in the calculated luminosity functions.

\section{Luminosity functions}

Figure 1 shows typical luminosity functions at three different observer frequencies. Overlaid on the numerical calculations are fits to the results using the function

$$
\phi(L)=C\left(\frac{L}{L_{0}}\right)^{-\lambda} \exp \left(-\frac{\ln \left(L / L_{0}\right)^{2}}{2 \sigma^{2}}\right) \exp \left(-\frac{L}{L_{0}}\right)
$$




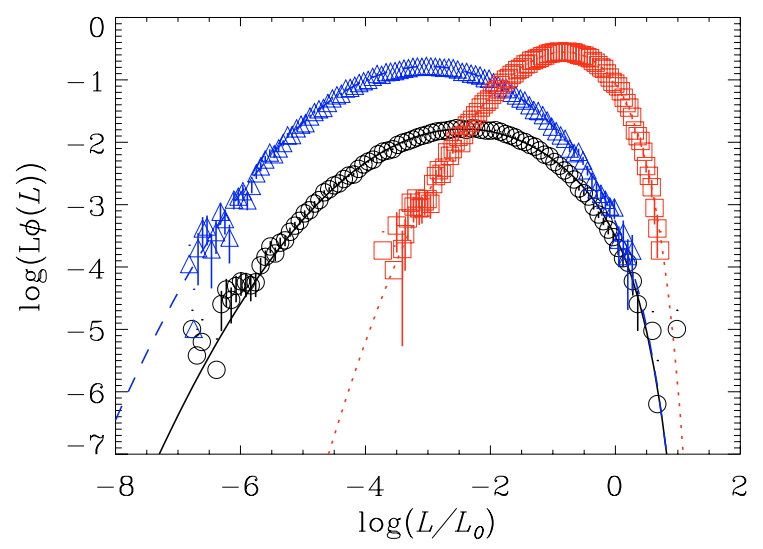

Fig. 1. Example luminosity functions obtained in our numerical calculations at three different frequencies: $2-10 \mathrm{keV}$ (circles, solid curve), $R$-band (triangles, dashed curve, blue in electronic edition) and $8.5 \mathrm{GHz}$ (squares, dotted curve, red in electronic edition). The curves are best fits using Eq. (1). For clarity we plot $L \phi(L) \times 0.1$ for $2-10 \mathrm{keV}$. Note that the general shape of the luminosity function is frequency independent although $\lambda, \sigma$ and $L_{0}$ are not.

We have chosen this function because of the parabolic resemblance of the plot shown in Fig. 1. The last exponential factor is needed since the luminosity function is not a pure parabola in $\log$-log space. Here, $L_{0}$ is a characteristic luminosity, defining an upper exponential cutoff in the function. Both $\sigma$ and $\lambda$ affect the width of the function, controlling its shape, while $\sigma$ has a slightly stronger effect. $C$ is a normalization constant and is determined by the other 3 parameters. Our results show that $\phi(L)$ is an accurate description of the numerical luminosity functions, giving a $\chi^{2}$ between 50 and 200 in each case for 100 degrees of freedom. Note that the function becomes a log-normal distribution with an exponential cutoff when $\lambda=1$. Our results show, however, that a value of $\lambda \approx 2$ is in the present case more likely, varying between the values 1.5 and 2.5 , depending on frequency and input model parameter distributions (see Fig. 3).

To investigate how the model parameter distributions affect our results, three different distribution shapes were tested, a uniform one, a Gaussian one and a triangular distribution with a maximum in the center of the parameter range. In the case of the Gaussian, the standard deviation was set at $1 / 4$ th the parameter range and the distribution restricted to the defined range. We found that the shape of the model parameter distributions did not change our general results, although the exact values of the parameters in Eq. (1) do depend on it. Since the function parameter values change only slightly between the chosen input distributions, we decided to use the triangular distribution for all our results below.

In addition to varying the shape of the model parameter distributions, we also tested how the parameter limits affect the outcomes. In each case, we changed the limits of only one parameter at a time. To cover all the basic effects, four tests were made: first we changed the upper limit keeping the lower limit fixed, next the lower limit was varied keeping the upper limit fixed, then the width was changed with a fixed center and finally the center was changed with a fixed width. The default values for the upper and lower limits are shown in Table 1. All of the parameters, except $p$, are distributed logarithmically and the width is then defined as the ratio between the upper and lower limit. Figure 2 shows the most interesting results from our tests. The parameters found from fitting the numerically calculated luminosity function with Eq. (1) are plotted against the parameter
Table 1. Default values for the upper and lower limit of the parameter ranges. All parameters except for $p$ are distributed logarithmically in the interval.

\begin{tabular}{lcc}
\hline \hline Parameter & Lower limit & Upper limit \\
\hline$E_{0}[\mathrm{erg}]$ & $10^{49}$ & $10^{51}$ \\
$n_{0}\left[\mathrm{~cm}^{-3}\right]$ & 0.1 & 10 \\
$\theta_{0}[\mathrm{deg}]$ & 2 & 15 \\
$p$ & 2.1 & 2.6 \\
$\epsilon_{\mathrm{e}}$ & 0.05 & 0.3 \\
$\epsilon_{\mathrm{B}}$ & $10^{-5}$ & $10^{-3}$ \\
\hline
\end{tabular}

that is being varied in each case. The general result from these tests, is that the function parameters $\lambda$ and $\sigma$ are not very dependent on the limits of the model parameter distributions, while $L_{0}$ can vary by several orders of magnitude. This allows us to conclude that the luminosity function can be presented by Eq. (1), setting both $\lambda \sim 2$ and $\sigma \sim 2$ and using $L_{0}$ as the only variable.

We see from Fig. 2 that the most significant model parameter is $E_{0}$, the initial energy release. Other model parameters that have significant effects on the parameters of $\phi(L)$ are $\theta_{0}$, the initial opening angle of the jet, and $\epsilon_{\mathrm{e}}$ and $\epsilon_{\mathrm{B}}$, the fractions of energy contained in the electron population and the magnetic field, respectively. The effects of the external density $n_{0}$ and the electron energy distribution index, $p$, on the function parameters are insignificant compared to the other model parameters.

The general trend when varying the parameter limits is that the value of $L_{0}$ is correlated with the upper limit (lower limit in case of $\theta_{0}$ ) of the model parameter range, while $\sigma$ is correlated with the width of the parameter distributions. This is shown, respectively, in the top and middle panel of Fig. 2. Note that the changes in the parameters of $\phi(L)$ depend in many cases strongly on the observed frequency. This can, for example, be seen in the value of $L_{0}$ while varying the upper limit of the $\epsilon_{\mathrm{e}}$. There are no effects in the radio waveband whereas the effects in optical and X-ray wavebands are similar and quite significant. Although the value of $\sigma$ depends most strongly on the width of the model parameter distribution, it can in some cases also depend on the center value of the model parameter distribution. The trend between the center value and $\sigma$ is, however, not as strong as the trend between $\sigma$ and the width.

The function parameter that shows the least dispersion when varying the limits of the model parameter distributions is $\lambda$. Its value is generally clustered around 2 and shows a greater dependency on frequency than on the model parameters, while its frequency dependence is in fact similar to that of $\sigma$ and $L_{0}$. Although $\lambda$ shows the least dispersion when varying the model parameter ranges, it is the parameter that is most difficult to constrain in the fitting procedure, resulting in the highest 1-sigma error. This can be seen in Fig. 3, which shows the value of $\sigma$ as a function of $\lambda$. It is also clear from the figure that there is an anti-correlation between the values of $\sigma$ and $\lambda$. Pearson's correlation test confirms this and results in correlation parameters between -0.5 to -0.8 depending on frequency. The correlation is strongest in the optical but weakest in the radio.

\section{Comparison with observations}

To compare our results with observations we took the recent luminosity compilation from Nardini et al. (2006) and converted it to luminosity functions using the same method as for our numerical results. This data is suitable for our use, since it is normalized to an observer's time of 1 day and a redshift of 1 . The 

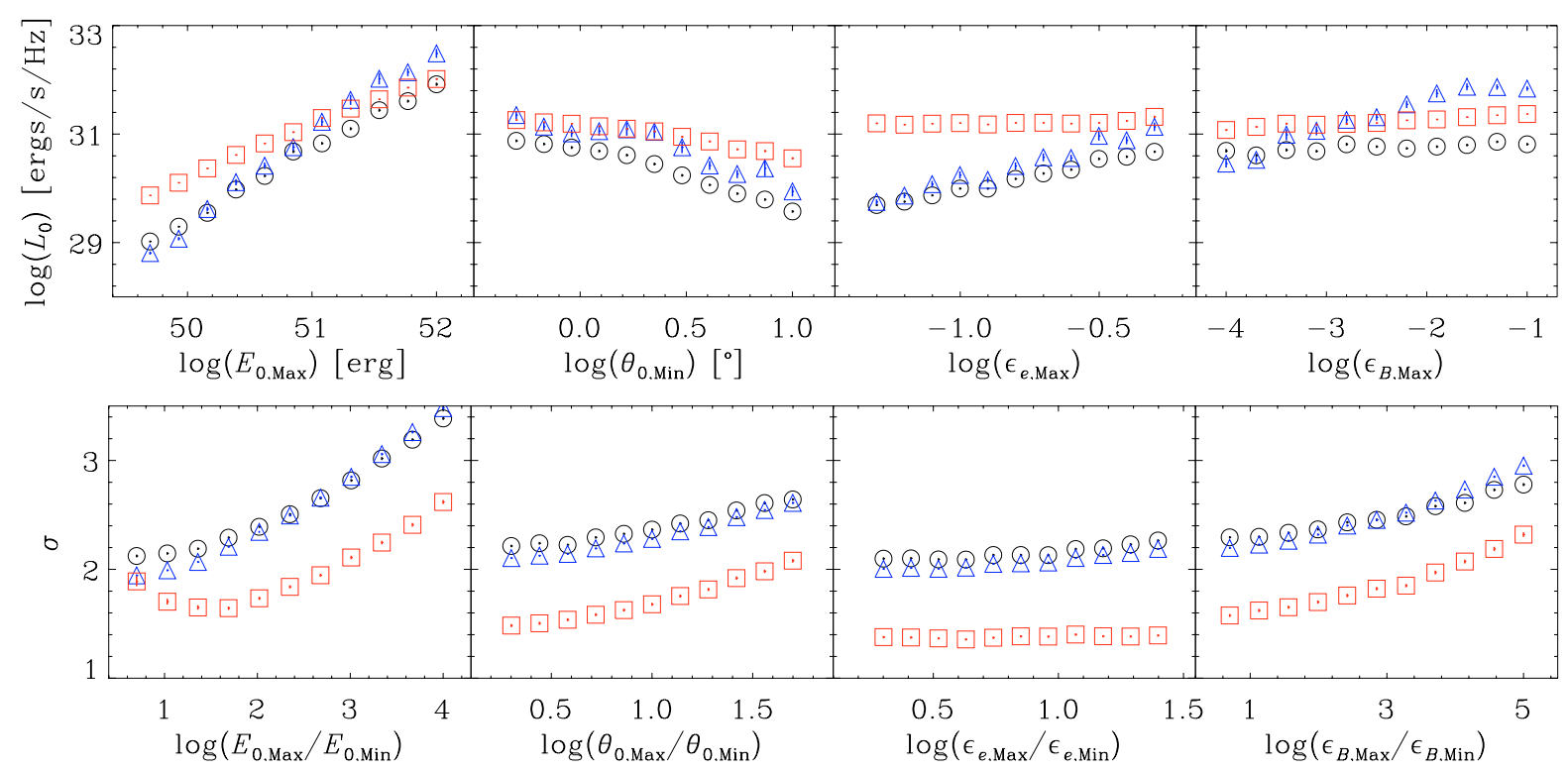

Fig. 2. Shown are the values of some of the function parameters found from fitting numerically calculated luminosity functions with Eq. (1). See Fig. 1 for legend. In each case we vary the limits of the model parameter distribution and show only the parameters that have the strongest effects. The default parameter limits are given in Table 1. Top panel: the value of $L_{0}$ while varying the upper limit (lower limit in the case of $\theta_{0}$ ) of the model parameter distribution keeping the lower limit (upper limit in the case of $\theta_{0}$ ) fixed. Shown are results for the parameters $E_{0}, \theta_{0}, \epsilon_{\mathrm{e}}$ and $\epsilon_{\mathrm{B}}$. We multiply $L_{0}$ by $10^{3}$ for $2-10 \mathrm{keV}$. Bottom panel: the value of $\sigma$ while varying the width of the model parameter distribution, keeping the center fixed. Shown are results for $E_{0}, \theta_{0}, \epsilon_{\mathrm{e}}$ and $\epsilon_{\mathrm{B}}$. Since the parameters are changed logarithmically, the width is the ratio between the upper and lower limit.

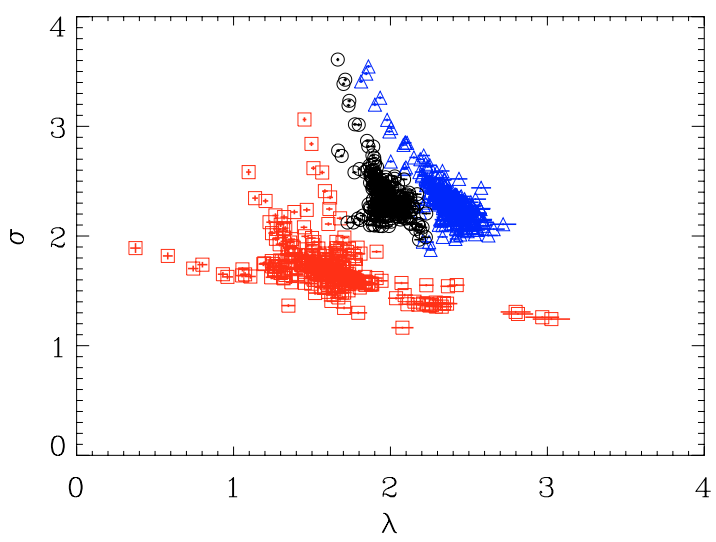

Fig. 3. The plot shows that there is an anti-correlation between the values of $\lambda$ and $\sigma$ found from fitting the numerically calculated luminosity functions with Eq. (1). The Pearson's correlation coefficient varies from -0.5 to -0.8 depending on frequency, being strongest in the optical range and weakest in radio. See Fig. 1 for legend.

data is displayed in Fig. 4 along with selected luminosity functions from our calculations. Note that our results are not formal fits to the observational data, but rather an eye-guided selection of one of our calculated luminosity functions. The sparse data set does not warrant a formal fit. Selection effects are also not taken into account for the same reason, but we refer interested readers to Nardini et al. (2007) which discusses the selection effects. The model parameter distribution that best resembles the observed X-ray luminosity function is the one with the center of the $E_{0}$ distribution at $10^{51} \mathrm{erg}$ but its width at the default value of 100 . Other parameters were at their default values, given in Table 1. This energy distribution is comparable to the one found from observations by Frail et al. (2001). The resulting luminosity function is, however, incompatible with the observed optical luminosity function that shows a clear over abundance of high

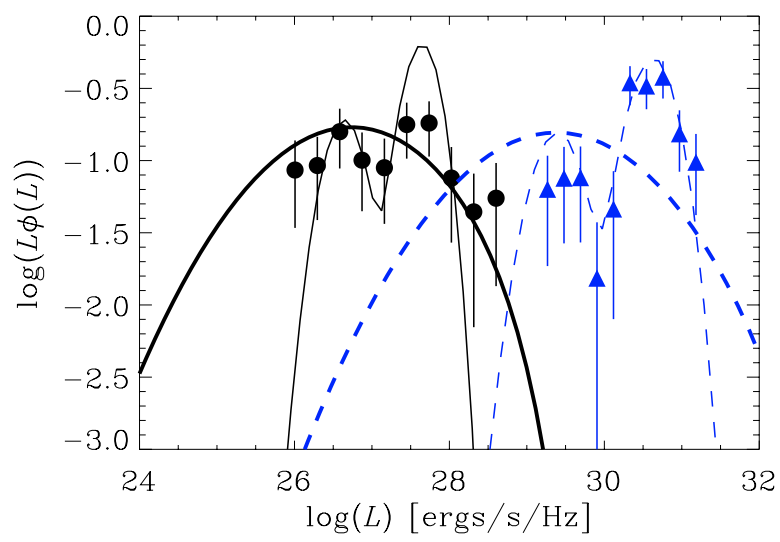

Fig. 4. Comparison between our numerical calculations (curves) and luminosity functions obtained from observational data (Nardini et al. 2006) (filled symbols). Two different models are shown, one wide (thick curves), assuming the value of the center of the $E_{0}$ distribution is at $10^{51} \mathrm{erg}$ and its width at the default value of 100 . Other parameters are as in Table 1. The other model is a composite luminosity function of two different populations, each with a much narrower parameter distribution (thin curves). Each population has, in this case, a different value for the center of the $E_{0}$ distribution and a different number of afterglows but are otherwise the same. See text for further discussion. Two different frequencies: $2-10 \mathrm{keV}$ (circles, solid curves) and $R$-band (triangles, dashed curves, blue in electronic edition) are shown.

luminosity afterglows which can not be explained by our results. There is also a sign of bimodality in the observed optical luminosity function (Liang \& Zhang 2006; Nardini et al. 2006; Kann et al. 2006; Jakobsson et al. 2006).

In an attempt to understand the optical luminosity function, we also created two afterglow populations with very narrow model parameter distributions, the width being a factor of 2 to 3 . These distributions are not compatible with parameter ranges found from fitting afterglow observations with the standard 
fireball model, where the ranges seem to be much larger. The two populations differ only in the value of the center of the $E_{0}$ distribution and the number of afterglows in each population. The more luminous population has the center value of $E_{0}$ at $10^{51} \mathrm{erg}$ while the fainter has the center at $2 \times 10^{50} \mathrm{erg}$. The number of afterglows in the more luminous population is four times higher than in the fainter one. Note that this ratio may change if observational biases are taken into account, as discussed below. These populations give two very narrow luminosity functions, which when added together agree with the observed optical luminosity function. It does not, however, seem to agree with the X-ray data, although a slight hint of a high luminosity peak may be seen. Its statistical significance is unknown at present.

There are a few possible explanations for the difference between the optical and X-ray luminosity functions. First, there may be two populations of afterglows with different parameter distributions, as shown by the thin lines in Fig. 4. Since this gives a good representation of the bimodal optical luminosity function and there is a hint of bimodality in the observed X-ray luminosity function, as discussed above, this explanation may seem to agree better with the observational data than a single population would. However, the narrow luminosity functions required for this explanation force the model parameter distributions to be much narrower than those found from afterglow modeling. Tight constraints between model parameters might, however, result in a similarly narrow luminosity functions and still comply with afterglow modeling. But even if that is the case, the ratio between the two peaks in each observed luminosity function is different and a fit with two afterglow populations would therefore be marginally good at best.

The second possibility is that the standard fireball model needs to be refined. Since there is a distinct difference between the optical and X-ray luminosity functions, a decoupling of the $\mathrm{X}$-ray and optical radiation mechanism may be needed. One example could be that the X-ray and optical radiation originate in different parts of the outflow. The bimodality of the optical luminosity function might then originate in two different populations of afterglows. The lack of bimodality in the X-ray luminosity function would then be accounted for by a larger dispersion in the radiation power of the X-ray zone. This possibility is also supported by recent observation of GRB afterglows (e.g. Perley et al. 2007; Oates et al. 2007) that show incompatibility with the standard fireball model because of differences between the X-ray and optical light curves.

Finally, the observed optical luminosity function may be incomplete. Limitations of current optical follow-up instruments, either in sensitivity or response time, lead to an observational bias for luminous afterglows. The bimodality is then expected to vanish with increasing number of observations. This may already have happened with the X-ray afterglow observations, as Gendre \& Boër (2005) found early on an evidence for bimodality in the X-ray luminosity function, which can now barely be seen with the more rapid and sensitive observations. If not real, the bimodality in the optical luminosity function may require a detection of a larger number of afterglows before it disappears, because of the redshift dependence of the data. The redshift is in most cases acquired from the optical afterglow spectrum and therefore requires an optically bright afterglow. This further increases the bias for optically bright afterglows without affecting the X-rays much. The recent study by Nardini et al. (2007) has shown that this is an unlikely explanation. The luminosity functions are, however, still based on a very small sample. In addition, there are also uncertainties involved when doing the $\mathrm{K}$-correction and time shift of the observational data. Hence, we cannot completely rule this explanation out.

\section{Discussion and conclusions}

In this letter we have shown, with the help of numerical calculations, that the luminosity functions of GRB afterglows can be described by a rather simple function given by Eq. (1). It is similar to a log normal distribution with an exponential cutoff. We have also shown that the shape of the luminosity function is not sensitive to the shape of the model parameter distributions. The function parameters are also quite robust, $\lambda$ and $\sigma$ vary by less than a factor of 2 , in most cases only by a few percent, for variations of orders of magnitude in the model parameters. It is mainly the upper cutoff, $L_{0}$, that is affected by the input parameters. This result can be used to get an approximate single parameter representation of the afterglow luminosity function. One must, however, bear in mind that $\sigma$ and $\lambda$ are somewhat dependent on our assumptions for the model parameter distributions. While we have chosen those to be representative of parameter estimates from current afterglow modeling, future modeling may require a re-evaluation of the values of $\lambda$ and $\sigma$. However, these are not expected to change much unless significant changes are seen in the model parameter distributions or strong correlations are found between some of the model parameters.

We have also shown that our results are in good general agreement with observations. However, incompatibility apparently exists between the optical and X-ray data, where a bimodality is seen in the optical luminosity function that is not apparent in the X-rays. The most likely explanation for this is that the standard fireball model is an incomplete description of the afterglow emission. A more detailed model or possibly an entirely different one is required, where a decoupling of the sources of X-ray and optical radiation is a natural ingredient. This is supported by recent observations, which have shown the afterglow behaviour to be more complex than expected (e.g. Nousek et al. 2006).

Acknowledgements. This work was partially supported by The Icelandic Research Fund and the University of Iceland Research Fund. We thank S. Courty for helpful discussions and P. Jakobsson for critical comments on an early version of the manuscript.

\section{References}

Costa, E., Frontera, F., Heise, J., et al. 1997, Nature, 387, 783

Frail, D. A., Kulkarni, S. R., Sari, R., et al. 2001, ApJ, 562, L55

Gendre, B., \& Boër, M. 2005, A\&A, 430, 465

Jakobsson, P., Fynbo, J. P. U., Ledoux, C., et al. 2006, A\&A, 460, L13

Jóhannesson, G., Björnsson, G., \& Gudmundsson, E. H. 2006a, ApJ, 640, L5

Jóhannesson, G., Björnsson, G., \& Gudmundsson, E. H. 2006b, ApJ, 647, 1238

Kann, D. A., Klose, S., \& Zeh, A. 2006, ApJ, 641, 993

Liang, E., \& Zhang, B. 2006, ApJ, 638, L67

Nardini, M., Ghisellini, G., Ghirlanda, G., et al. 2006, A\&A, 451, 821

Nardini, M., Ghisellini, G., \& Ghirlanda, G. 2007, to be published

Nousek, J. A., Kouveliotou, C., Grupe, D., et al. 2006, ApJ, 642, 389

Oates, S. R., De Pasquale, M., Page, M. J., et al. 2007, ArXiv e-prints Panaitescu, A. 2005, MNRAS, 363, 1409

Panaitescu, A., \& Kumar, P. 2001, ApJ, 554, 667

Perley, D. A., Bloom, J. S., Butler, N. R., et al. 2007, ArXiv Astrophysics e-prints van Paradijs, J., Groot, P. J., Galama, T., et al. 1997, Nature, 386, 686 